\title{
Análise e caracterização do panorama da distribuição de Cirurgiões-Dentistas no Brasil
}

\author{
Analysis and characterization of the dentist's distribution overview in Brazil
}

\section{Análisis y caracterización del panorama de la distribución de odontólogos en Brasil}

\section{Ernani Canuto FIGUEIRÊDO JÚNIOR ${ }^{1}$}

Nathália Curvêlo UCHÔA ${ }^{2}$

Jozinete Vieira PEREIRA

${ }^{1}$ Doutorando em Odontologia - Programa de Pós-graduação em Odontologia, Universidade Estadual da Paraíba, UEPB 58429-500 Campina Grande-PB, Brasil ${ }^{2}$ Graduanda em Odontologia,Universidade Estadual da Paraíba, UEPB 58223-000 Araruna - PB, Brasil

${ }^{3}$ Professora do Programa de Pós-graduação em Odontologia, Universidade Estadual da Paraíba, 58429-500 Campina Grande-PB, Brasil

\section{Resumo}

Dentre a categoria odontológica, Cirurgiões-Dentistas (CDs) são os únicos profissionais que tem atribuição exclusiva de prestar assistência clínica direta ao público de forma independente, apresentando um papel importante na Odontologia. O objetivo deste trabalho é caracterizar o panorama da distribuição de CDs no Brasil, considerando-a entre estes profissionais em si e entre a população brasileira, apresentando considerações sobre o perfil profissional e mercadológico dos CDs. O trabalho consiste em um levantamento de dados acerca dos números de CDs no Brasil e do número de habitantes da população brasileira. Os dados mostram que existem 309.088 CDs em exercício no Brasil, os quais concentram-se principalmente nos estados de São Paulo, Minas Gerais, Rio de Janeiro e Paraná. Em nível nacional, há 1CD para 617,1 habitantes.Dentre os estados, Distrito Federal, São Paulo, Santa Catarina, Rio de Janeiro e Paraná apresentam os menores valores proporcionais. As regiões brasileiras Sudeste e Sul concentram os maiores percentuais de CDs do país, enquanto o Norte possui o menor percentual. Paralelamente, Sudeste e Centro-Oeste apresentam as menores proporções de CD/habitantes, enquanto o Nordeste apresenta a maior razão dessa distribuição. Os resultados apontam o atual perfil de distribuição profissional e mercadológica de CDs no Brasil, evidenciando disparidades entre as diferentes localidades do país, podendo assim representar um indicador importante acerca do planejamento mercadológico para os CDs, bem como fornecer reflexões acerca da discrepância entre a quantidade de CDs e o quadro de saúde bucal ainda presente no país.

Descritores: Censos; Odontólogos; Distribuição Espacial da População; Recursos Humanos em Odontologia; Mercado de Trabalho.

\section{Abstract}

Among the dental category, dentists are the only professionals that have exclusive attribution of providing direct clinical assistance to the public independently, presenting an important prominence in Dentistry. The aim of this paper is to characterize the dentists' distribution overview in Brazil, considering it among these professionals in them and among the Brazilian population, presenting considerations in the job market. The paper consists of a survey of data about the numbers of dentists in Brazil and the number of inhabitants of the Brazilian population. The data show that there are 309.088 practicing dentists in Brazil, which are mainly concentrated in the states of São Paulo, Minas Gerais, Rio de Janeiro and Paraná. At the national level, there is 1 dentist for 617,1 inhabitants. Among the states, the Federal District, São Paulo, Santa Catarina, Rio de Janeiro and Paraná present the lowest proportional values. The Brazilian regions Southeast and South concentrate the largest percentage of dentists in the country, while the North has the lowest percentage. At the same time, the Southeast and Center-West have the lowest proportions of dentists/inhabitants, while the Northeast has the highest ratio of this distribution. The results show the current profile and job market of these professionals in Brazil, evidencing disparities between the different localities in the country, thus being able to represent an important indicator about market planning for dentists, as well as to provide reflections on the discrepancy between the quantity of dentists and the oral health situation still present in the country.

Descriptors: Censuses; Dentists; Residence Characteristics; Dental Staff; Job Market.

\section{Resumen}

Entre la categoría odontológica, los odontólogos son los únicos profesionales que tienen atribución exclusiva de prestar asistencia clínica directa al público de forma independiente, presentando un papel importante en la Odontología. El objetivo de este trabajo es caracterizar el panorama de la distribución de odontólogos en Brasil, considerándola entre estos profesionales en sí y entre la población brasileña, presentando consideraciones sobre el perfil profesional y mercadológico de los mismos. El trabajo consiste en un levantamiento de datos sobre los números de odontólogos en Brasil y del número de habitantes de la población brasileña. Los datos muestran que existen 309.088 odontólogos en ejercicio en Brasil, los cuales se concentran principalmente en los estados de São Paulo, Minas Gerais, Río de Janeiro y Paraná. A nivel nacional, hay 1 odontólogo para 617,1 habitantes. En los estados, Distrito Federal, São Paulo, Santa Catarina, Río de Janeiro y Paraná presentan los menores valores proporcionales. Las regiones brasileñas Sudeste y Sur concentran los mayores porcentuales de odontólogos del país, mientras que el Norte tiene el menor porcentaje. Paralelamente, Sudeste y Centro-Oeste presentan las menores proporciones de odontólogos/habitantes, mientras que el Nordeste presenta la mayor razón de esa distribución. Los resultados apuntan al actual perfil de distribución profesional y mercadológica de odontólogos en Brasil, evidenciando disparidades entre las diferentes localidades del país, pudiendo así representar un indicador importante acerca del planeamiento mercadológico, así como proporcionar reflexiones acerca de la discrepancia entre la cantidad de profesionales y el cuadro de salud bucal todavía presente en el país.

Descriptores: Censos; Odontólogos; Distribución Espacial de la Población; Personal de Odontología; Mercado de Trabajo.

\section{INTRODUÇÃO}

A Odontologia é uma área de atuação profissional que se exerce em benefício da saúde do ser humano, da coletividade e do meio ambiente, tendo a saúde do ser humano como objetivo de atenção ${ }^{1}$.

O exercício da Odontologia é regido no âmbito do território brasileiro de acordo com a Lei $\mathrm{n}^{\circ}$ 5.081, regulamentada em 24 de Agosto de $1966^{2}$, observando-se em legislações como a Resolução CFO 63/2005 $5^{3}$ e atualizações como a Resolução CFO 160/2015 ${ }^{4}$ e Resolução CFO 161/2015 5 a definição de aspectos regulamentadores acerca do exercício profissional das diferentes categorias odontológicas e demais aspectos relacionados à Odontologia no Brasil e atividades vinculadas.

Dentre os profissionais que compõem as categorias odontológicas, os Cirurgiões-Dentistas (CDs) apresentam um papel importante, uma vez que conforme a Resolução CFO 63/2005 ${ }^{3}$ são os únicos que possuem a atribuição exclusiva de prestar assistência clínica direta ao público de forma independente. Embora outras categorias profissionais 
como Técnicos em Saúde Bucal e Auxiliares em Saúde Bucal possam prestar assistência direta ou indireta a pacientes, conforme a Resolução CFO $63 / 2005^{3}$ e a Lei n ${ }^{\circ} 11.889$, regulamentada em 24 de Dezembro de $2008{ }^{6}$, a atuação desses profissionais para esse tipo de atividade está condicionada e dependente da supervisão dos CDs.

Assim, percebe-se a importância e o papel dos CDs no exercício da profissão odontológica através da prestação de seus serviços e no desenvolvimento das atividades de sua competência, tanto no setor público quanto no setor privado ${ }^{7}$.

Nessa perspectiva, o objetivo deste trabalho foi caracterizar o panorama da distribuição de CDs no Brasil, considerando a distribuição desses profissionais entre si e entre a população brasileira, apresentando e trazendo considerações acerca do perfil profissional e mercadológico dos CDs em âmbito nacional.

\section{MATERIAL E MÉTODO}

O presente trabalho consiste em estudo realizado através de técnica de documentação indireta, por meio do levantamento de dados realizado em documentos escritos acessados em arquivos públicos e fontes estatísticas ${ }^{8}$.

Os dados apresentados consistiram no levantamento da distribuição quantitativa e percentual do número de CDs no Brasil, assim como na caracterização do perfil da distribuição quantitativa de CDs por habitantes, com base nos dados referentes à população brasileira. Para a categorização dos dados, ambos os aspectos foram considerados tendo como parâmetro a utilização dos critérios de regionalização por estados, regiões e pela federação brasileira. Os resultados estão apresentados através de estatística descritiva.

A metodologia da coleta de dados baseou-se de acordo com a abordagem adotada em investigações realizadas em estudos preliminares ${ }^{9-12}$ de temática semelhante à do presente estudo. Para tal, as informações referentes ao número de $\mathrm{CDs}$ foram coletadas diretamente através do site do Conselho Federal de Odontologia (CFO) $)^{13}$, considerando o número total de CDs cadastrados e ativos no Brasil, de acordo com a distribuição geográfica por Unidades da Federação e regiões do país. Por outro lado, o número indivíduos referentes à população brasileira foi coletado diretamente através do site do Instituto Brasileiro de Geografia e Estatística $(\mathrm{IBGE})^{14}$ tomando como base o número de indivíduos da população das Unidades da Federação e do Brasil, como base no último censo demográfico brasileiro, realizado em 2010. Ambos os dados são de domínio e acesso público.

As pesquisas do quantitativo de CDs foi realizada no dia 24 de Agosto de 2018 no site do $\mathrm{CFO}^{13}$, consistindo no número exato de profissionais devidamente regulamentados no conselho profissional até as datas das buscas. Já a pesquisa populacional foi realizada também no dia 24 de Agosto de 2018 no site do IBGE $^{14}$, consistindo no número exato da população de cada Unidade da Federação e a população total do Brasil.

\section{RESULTADOS}

Até a data da realização da pesquisa, o total de 309.088 CDs que estava devidamente cadastrado no CFO e em exercício no Brasil está apresentado na Tabela 1. Com base na regionalização brasileira em Unidades da Federação, os estados nos quais o maior número desses profissionais exercem suas atividades são respectivamente São Paulo, Minas Gerais, Rio de Janeiro e Paraná, que representam 29,56\%; 11,68\%; $10,15 \%$ e $6,29 \%$ do total de CDs do país. Por outro lado, o Acre é o estado brasileiro que possui o menor número de $\mathrm{CDs}(\mathrm{n}=807)$, representados por apenas $0,26 \%$ do total de profissionais do Brasil.

Tabela 1. Número e distribuição quantitativa e percentual de CDs ativos e de habitantes por Unidade da Federação e relação entre a quantidade de $\mathrm{CD} /$ número de habitantes por Unidade da Federação

\begin{tabular}{|c|c|c|c|c|c|}
\hline $\begin{array}{l}\text { Unidades da } \\
\text { Federacão }\end{array}$ & CDs ativos ${ }^{*}$ & $\begin{array}{l}\text { \% CDs } \\
\text { ativos }\end{array}$ & Habitantes** & $\begin{array}{c}\% \\
\text { habitantes }\end{array}$ & $\begin{array}{c}\text { Relação } \\
\text { CD/habitantes }\end{array}$ \\
\hline Acre & 807 & $0,26 \%$ & 733.559 & $0,38 \%$ & $1 / 908,9$ \\
\hline Alagoas & 3.017 & $0,98 \%$ & 3.120 .494 & $1,64 \%$ & $1 / 1.034,3$ \\
\hline Amapá & 861 & $0,28 \%$ & 669.526 & $0,35 \%$ & $1 / 777,6$ \\
\hline Amazonas & 4.372 & $1,41 \%$ & 3.483 .985 & $1,83 \%$ & $1 / 796,8$ \\
\hline Bahia & 12.955 & $4,19 \%$ & 14.016.906 & $7,35 \%$ & $1 / 1.081,9$ \\
\hline Ceará & 7.180 & $2,32 \%$ & 8.452 .381 & $4,43 \%$ & $1 / 1.177,2$ \\
\hline $\begin{array}{l}\text { Distrito } \\
\text { Federal }\end{array}$ & 7.307 & $2,36 \%$ & 2.570 .160 & $1,35 \%$ & $1 / 351,7$ \\
\hline $\begin{array}{l}\text { Espírito } \\
\text { Santo }\end{array}$ & 5.818 & $1,88 \%$ & 3.514 .952 & $1,84 \%$ & $1 / 604,1$ \\
\hline Goiás & 10.693 & $3,46 \%$ & 6.003 .788 & $3,15 \%$ & $1 / 561,4$ \\
\hline Maranhão & 4.408 & $1,43 \%$ & 6.574 .789 & $3,45 \%$ & $1 / 1.491,5$ \\
\hline Mato Grosso & 4.834 & $1,56 \%$ & 3.035 .122 & $1,59 \%$ & $1 / 627,8$ \\
\hline $\begin{array}{l}\text { Mato Grosso } \\
\text { do Sul }\end{array}$ & 4.219 & $1,36 \%$ & 2.449 .024 & $1,28 \%$ & $1 / 580,4$ \\
\hline $\begin{array}{l}\text { Minas } \\
\text { Gerais }\end{array}$ & 36.098 & $11,68 \%$ & 19.597 .330 & $10,27 \%$ & $1 / 542,8$ \\
\hline Pará & 5.473 & $1,77 \%$ & 7.581 .051 & $3,97 \%$ & $1 / 1.385,1$ \\
\hline Paraíba & 4.739 & $1,53 \%$ & 3.766 .528 & $1,97 \%$ & $1 / 794,7$ \\
\hline Paraná & 19.427 & $6,29 \%$ & 10.444 .526 & $5,48 \%$ & $1 / 537,6$ \\
\hline Pernambuco & 8.671 & $2,81 \%$ & 8.796 .448 & $4,61 \%$ & $1 / 1.014,4$ \\
\hline Piauí & 3.112 & $1,01 \%$ & 3.118 .360 & $1,63 \%$ & $1 / 1.002,0$ \\
\hline $\begin{array}{l}\text { Rio de } \\
\text { Janeiro }\end{array}$ & 31.378 & $10.15 \%$ & 15.989 .929 & $8,38 \%$ & $1 / 509,5$ \\
\hline $\begin{array}{l}\text { Rio Grande } \\
\text { do Norte }\end{array}$ & 3.887 & $1,26 \%$ & 3.168 .027 & $1,66 \%$ & $1 / 815,0$ \\
\hline $\begin{array}{l}\text { Rio Grande } \\
\text { do Sul }\end{array}$ & 18.543 & $6,00 \%$ & 10.693 .929 & $5,61 \%$ & $1 / 576,7$ \\
\hline Rondônia & 2.239 & $0,72 \%$ & 1.562 .409 & $0,82 \%$ & $1 / 697,8$ \\
\hline Roraima & 819 & $0,26 \%$ & 450.479 & $0,24 \%$ & $1 / 550,0$ \\
\hline $\begin{array}{l}\text { Santa } \\
\text { Catarina }\end{array}$ & 12.645 & $4,09 \%$ & 6.248 .436 & $3,28 \%$ & $1 / 494,1$ \\
\hline São Paulo & 91.376 & $29,56 \%$ & 41.262 .199 & $21,63 \%$ & $1 / 451,5$ \\
\hline Sergipe & & $0,67 \%$ & 2.068 .017 & $1,08 \%$ & $1 / 998,0$ \\
\hline Tocantins & & $0,69 \%$ & 1.383 .445 & $0,73 \%$ & $1 / 647,0$ \\
\hline BRASIL & 309.088 & $100,00 \%$ & 190.755 .799 & $100,00 \%$ & $1 / 617,1$ \\
\hline
\end{tabular}

*De acordo com dados cadastrados no site do CFO. Fonte: Sistema de Cadastro - Rotina SISGER02 - (24/08/2018 05:42 h)

** De acordo com dados cadastrados no site do IBGE

Tendo por base a população de cada um dos estados brasileiros, percebe-se que São Paulo, Minas Gerais, Rio de Janeiro e Bahia são respectivamente os estados mais populosos do país, e os três primeiros estados, concentram respectivamente o maior número de CDs do Brasil, como já mencionado.

Em outro extremo, embora Roraima seja o estado menos populoso do Brasil, com apenas 0,24\% do total da população brasileira, possui uma quantidade de CDs $(\mathrm{n}=819)$ maior que o encontrado no estado do Acre $(\mathrm{n}=807)$.

Considerando-se a relação entre os números de CDs por habitantes, observa-se que os valores 
encontrados para o Brasil foram da ordem de $1 \mathrm{CD}$ para 617,1 habitantes. Enquanto isso, considerandose as Unidades da Federação, o Distrito Federal possui a menor distribuição de profissionais por habitantes, com uma proporção de $1 \mathrm{CD}$ para 351,7 pessoas, representando a melhor razão de distribuição proporcional de $\mathrm{CD}$ por habitantes.

As demais posições iniciais dentre o ranking dos estados que apresentam as menores proporções CDs/habitantes são encontradas em São Paulo, Santa Catarina, Rio de Janeiro e Paraná, com proporções de 1 CD para cada 451,5; 494,1; 509,5 e 537,6 habitantes, respectivamente.

Dentro dessa perspectiva, a despeito de o estado de São Paulo reunir o maior número quantitativo e proporcional de CDs do país, observa-se que o mesmo não representa a Unidade da Federação em que há a melhor distribuição de profissionais/número de habitantes, ficando nesse aspecto, em segundo lugar dentro desse ranking.

Por outro lado, os estados do Rio de Janeiro e Paraná representam respectivamente a terceira e quarta posição dentre os estados com os maiores quantitativos e percentuais de CDs do país, ocupando respectivamente a quarta e quinta posição dentre o ranking de estados com as menores taxas de distribuição de profissionais/número de habitantes.

Em outro extremo, para esse mesmo aspecto, a maior proporção $\mathrm{CD} /$ habitantes foi encontrada no estado do Maranhão, possuindo um total de $1 \mathrm{CD}$ para cada 1.491,5 habitantes. Enquanto que seguindo-se a este, os estados do Pará, Ceará e Bahia também apresentam as posições extremas dessa proporção.

De acordo com a distribuição dos profissionais de acordo com os critérios de divisão pelas regiões brasileiras (Tabela 2), verifica-se que o Sudeste representa a região mais populosa, concentrando também o maior percentual de CDs do país, abrangendo mais da metade dentre o total de profissionais em atividade. Seguindo-se a ordem de concentração de CDs, o Sul representa a segunda região onde há a maior concentração $(16,38 \%)$ do número total de profissionais ( $\mathrm{n}=50.615)$, abrangendo $16,38 \%$ do total de profissionais do país. Os valores encontrados para a região Sul apresentam-se próximos àqueles encontrados para o Nordeste, que embora mais populoso que o Sul, apresenta um número menor de CDs, com uma diferença de 574 profissionais a menos que esta última.

Por outro lado, o menor quantitativo e percentual de profissionais encontra-se na região Norte, com apenas 5,41\% do total de CDs brasileiros. Mais uma vez, considerando-se a relação entre o número de CDs por habitantes, observa-se que dentre as regiões brasileiras, o menor valor é igualmente encontrado no Sudeste, seguindo-se da região CentroOeste, que embora seja a região menos populosa, possui uma distribuição profissional/habitantes da ordem de 1 CD para cada 519,6 habitantes. Valores limítrofes para esta relação são encontrados no Nordeste, que embora represente a terceira posição dentre as regiões com maior número de CDs, apresenta a maior razão na distribuição de CDs por habitantes.

Tabela 2- Número e distribuição quantitativa e percentual de CDs ativos e de habitantes por região brasileira e relação de $\mathrm{CD} /$ número de habitantes por região brasileira

\begin{tabular}{|c|c|c|c|c|c|}
\hline $\begin{array}{l}\text { Região } \\
\text { geográfica }\end{array}$ & $\underset{\text { ativos }}{\text { CDs }}$ & $\begin{array}{l}\text { \% CDs } \\
\text { ativos }\end{array}$ & Habitantes** & $\begin{array}{c}\% \\
\text { habitantes }\end{array}$ & $\begin{array}{c}\text { Relação } \\
\text { CD/ } \\
\text { habitantes }\end{array}$ \\
\hline $\begin{array}{l}\begin{array}{l}\text { Centro- } \\
\text { oeste }\end{array} \\
\end{array}$ & 27.053 & $8,75 \%$ & 14.058 .094 & $7,37 \%$ & $1 / 519,6$ \\
\hline Nordeste & 50.041 & $16,19 \%$ & 53.081 .950 & $27,83 \%$ & $1 / 1.060,7$ \\
\hline Norte & 16.709 & $5,41 \%$ & 15.854 .454 & $8,31 \%$ & $1 / 948,8$ \\
\hline Sudeste & 164.670 & $53,28 \%$ & 80.364 .410 & $42,13 \%$ & $1 / 488,0$ \\
\hline Sul & 50.615 & $16,38 \%$ & 27.386 .891 & $14,36 \%$ & $1 / 541,0$ \\
\hline BRASIL & 309.088 & $100,00 \%$ & 190.755 .799 & $100,00 \%$ & $1 / 617,1$ \\
\hline
\end{tabular}

\section{DISCUSSÃO}

O exercício da profissão odontológica analisado sob a ótica do contexto mundial ${ }^{15}$ e nacional $^{16}$ apresenta uma dinâmica historicamente caracterizada pela sua evolução como categoria profissional, desde os seus primórdios enquanto profissão não regulamentada até sua configuração como profissão devidamente regulamentada ${ }^{15,16}$.

Paralelamente, essa transformação também pôde ser atribuída ao perfil profissional e mercadológico ${ }^{15}$, baseados sobretudo em decorrência das demandas sociais através da evolução das políticas públicas voltadas para a resolução dos agravos bucais assim como das demandas do serviço odontológico privado $^{12}$.

Dentro dessa perspectiva, considerando-se o contexto brasileiro, a evolução e crescimento quantitativo no número de profissionais ${ }^{12} \mathrm{em}$ associação com as transformações observadas em modelos assistenciais tais como os da saúde pública $^{17}$ trouxeram mudanças no mercado odontológico ${ }^{12}$ e no direcionamento e organização dos serviços em saúde bucal ${ }^{17}$. Nesse aspecto, os dados evidenciados pelo presente estudo refletem a atual conjuntura acerca do perfil de distribuição de CDs no Brasil, representados pela caracterização de como esses profissionais estão alocados entre as distintas localidades do país. Além disso, esses dados apresentam uma atualização acerca das informações existentes em investigações anteriormente realizadas com o mesmo objetivo $^{9,10,12,18}$.

Esses achados também apontam e corroboram aspectos já evidenciados na literatura, notadamente no que se refere às disparidades entre a quantidade e distribuição de profissionais entre as diferentes localidades do país ${ }^{9,10,12,18,19}$, principalmente em regiões como Sudeste e Sul ${ }^{12,18,19}$. Desse modo, reflete os cenários distintos 
acerca da distribuição de profissionais entre as regiões brasileiras, evidenciando um perfil de maior saturação de CDs para determinadas regiões, bem como uma maior escassez em outras ${ }^{12}$.

Paralelamente a isso, os achados ora apresentados confirmam que o incremento do quantitativo de CDs em exercício no país consiste em uma realidade apontada por outros estudos ${ }^{12}$, refletindo a constante tendência de evolução acerca do mercado de trabalho para essa categoria profissional. Nesse aspecto, compreender como os CDs estão distribuídos no país pode representar um importante indicador de planejamento profissional sob o ponto de vista mercadológico, considerando-se, principalmente, aspectos como a saturação de profissionais em determinadas regiões, aspecto esse já relatado pela literatura ${ }^{12}$ e aqui mais uma vez caracterizado, evidenciando a sua constante evolução.

Por outro lado, sob o ponto de vista epidemiológico, o conhecimento desses dados pode fornecer um perfil e reflexões acerca da situação de saúde bucal esperada pelo país. Esse aspecto pode ser exemplificado através do conhecimento da proporção de CDs existente por habitantes. De acordo com o preconizado pela Organização Mundial da Saúde (OMS), um valor equivalente a proporção de um CD para 1.500 habitantes apresentaria suficiência para 0 atendimento das demandas de saúde bucal da população $^{10,12}$. Considerando-se esse contexto, os dados do presente estudo evidenciam que nenhuma Unidade da federação possui um número de CDs por habitantes menor que o recomendado, apresentando assim um número de CDs maior que o necessário. Além disso, em âmbito nível nacional esse valor apresenta-se igualmente satisfatório, corroborando os achados de investigações prévias ${ }^{12}$, e apresentando-se atualmente como 2,43 vezes maior que o preconizado.

Sob outro aspecto, considerando esses valores por regiões brasileiras, embora nenhuma delas apresente uma quantidade de CDs inferior à preconizada, o Nordeste e o Norte possuem as piores razões de distribuição de CDs por habitantes, ao passo que as regiões Sudeste, Centro-Oeste e Sul possuem os perfis mais favorecidos. Embora esses valores estejam ora atualizados, os mesmos corroboram uma realidade já apresentada por estudo anterior $^{12}$, demonstrando que todas as regiões brasileiras apresentam, no momento atual, um número de $\mathrm{CDs}$ maior do que o recomendado pela OMS.

Em outra instância, considerando-se que os resultados de levantamentos epidemiológicos nacionais representativos como a Pesquisa Nacional de Saúde Bucal - Projeto SB Brasil 2010 20 evidenciam a presença de discrepâncias na situação de saúde bucal entre as diferentes regiões brasileiras, o atual cenário de distribuição de CDs/habitantes permite corroborar e estabelecer reflexões acerca das discrepâncias entre o quantitativo efetivo de CDs e o quadro de saúde bucal do Brasil, que embora observada anteriormente ${ }^{21}$, permanece existindo no contexto Brasil.

\section{CONCLUSÃO}

A distribuição quantitativa de $\operatorname{CDs}$ e a de CDs por habitantes de acordo com a distribuição populacional brasileira revela que em determinados estados e regiões do Brasil há uma saturação de profissionais, enquanto que outros apresentam relativa escassez dos mesmos, apontando possíveis áreas ou regiões para as quais o mercado de trabalho apresenta maior ou menor potencial promissor, podendo, portanto fornecer aspectos $\mathrm{e}$ indícios orientadores tanto aos CDs já existentes quanto os futuros CDs acerca de indicadores de planejamento profissional e mercadológico.

\section{REFERENCIAS}

1. Brasil. Conselho Federal de Odontologia. Código de Ética Odontológica 2015. [Internet] Citado em 2018 ago 25.Disponível em: < http://cfo.org.br/ website/wp-content/uploads/ 2018/03/ codigo_ etica.pdf >

2. Brasil.Presidência da República.Casa civil. Lei ${ }^{\circ}$ 5.081 de 24 de agosto de 1966. [Internet] Citado em 2018 ago 25. Disponível em: < http: //www.planalto.gov.br/ccivil_03/leis/L5081.htm>

3. Brasil. Conselho Federal de Odontologia. Resolução CFO 63/2005. [Internet] Citado em 2018 ago 25. Disponível em: http://cfo.org.br/website/ wp-content/ uploads/ 2018/03/consolidacao.pdf

4. Brasil. Conselho Federal de Odontologia. Resolução CFO 160/2015. [Internet] Citado em 2018 ago 25.Disponível em:< http://www.cfo.org.br/ website/ wp-content/ uploads/2015/11/Resolu\%C3\%A7\%C3\%A3oCFO-160-15-novas-especialidades.pdf $>$

5. Brasil. Conselho Federal de Odontologia. Resolução CFO 161/2015. [Internet] Citado em 2018 ago 25. Disponível em: <http://www.cfo. org.br/ website/ wpcontent/ uploads /2015/11/ Resolu\%C3\% A7\%C3\%A3o- CFO-161-15- novaespecialidade-II.pdf>

6. Brasil.Presidência da República.Casa civil. Lei $n^{\circ}$ 11.889 de 24 de dezembro de 2008. [Internet] Citado em 2018 ago 25.Disponível em: < http://www.planalto.gov.br/ccivil_03/_Ato20072010/2008/Lei/L11889.htm>

7. Reis WG, Scherer MDA, Carcereri DL. O trabalho do cirurgião-dentista na Atenção Primária à Saúde: entre o prescrito e o real. Saúde debate. 2015;39(104):56-64.

8. Lakatos EM, Marconi MM. Técnicas de pesquisa. 7.ed. São Paulo: Atlas; 2008

9. Paranhos LR, Ricci ID, Siqueira DF, Scanavini 
MA, Daruge Júnior E. Análise do mercado de trabalho odontológico na região Nordeste do Brasil. Rev Odontol Univ Cid São Paulo. 2009;21(2):104-18.

10.Paranhos LR, Ricci ID, Almeida Filho RP, Castro R, Scanavini MA. Análise do mercado de trabalho odontológico na região norte do Brasil. Rev Odontol Univ Cid São Paulo. 2009;17(34):27-36.

11.Fernandes Neto JA, Silva AMT, Catão MHCV. Odontogeriatras, geriatras e idosos brasileiros: uma análise por estados e regiões do país. Arch Health Invest. 2016;5(5):262-66.

12.San Martin AS, Chisini LA, Martelli S, Sartori LRM, Ramos EC, Demarco FF. Distribuição dos cursos de Odontologia e de cirurgiões-dentistas no Brasil: uma visão do mercado de trabalho. Rev ABENO. 2018;18(1):63-73.

13.CFO: Conselho Federal de Odontologia. [Internet]. Citado em 2018 ago 24. Disponível em:<http://cfo.org.br/website/ >

14.IBGE: Instituto Brasileiro de Geografia e Estatística [Internet]. 2010. Citado em 2018 ago 24. Disponível em: < https://cidades.ibge. gov.br/brasil/panorama>

15.Ferreira NA, Ferreira AP, Freire, MCM. Mercado de trabalho na Odontologia: contextualização e perspectivas. Rev.Odontol. UNESP. 2013; 42(4):304-9.

16.Pereira W. Uma história da Odontologia no Brasil. Histórias e perspectivas. 2012;47(1):147-73.

17. Brasil. Ministério da Saúde. Secretaria de Atenção à Saúde. Departamento de Atenção Básica. Saúde Bucal. Cadernos de Atenção Básica $\mathrm{n}^{\circ} 17$. Brasília: Ministério da Saúde; 2008. Disponível em: http://bvsms.saude.gov. br/bvs/publicacoes/ saude_bucal.pdf

18. Morita MC, Haddad AE, Araújo ME. Perfil atual e tendências do cirurgião-dentista brasileiro. Maringá: Dental Press International; 2010.

19.Junqueira CL, Ramos DLP, Rode SM. Considerações sobre o mercado de trabalho em Odontologia. Rev Paul Odontol. 2005; 26(4):24-7.

20.Brasil. Ministério da Saúde. Secretaria de Atenção à Saúde. Secretaria de Vigilância em Saúde. SB Brasil 2010: Pesquisa Nacional de Saúde Bucal: resultados principais. Brasília: Ministério da Saúde; 2012. 116 p.

21. Sato FRL. Mercado de Assistência Odontológica Suplementar: situação atual e perspectivas futuras. Rev Assoc Paul Cir Dent. 2005;59(1):37-41.
CONFLITO DE INTERESSES

Os autores declaram não haver conflitos de interesse.

\section{AUTOR PARA CORRESPONDENCIA}

\section{Ernani Canuto Figueirêdo Júnior}

ernanicfjunior@outlook.com

Submetido em 29/08/2018

Aceito em 04/10/2018 